\title{
Effect of guide vane on pressure fluctuation and radial force in hydraulic turbine
}

\author{
Fengxia Shi ${ }^{1}$ and Ningrui Zhang ${ }^{2, a}$ \\ ${ }^{1}$ School of Energy and Power Engineering, Lanzhou University of Technology, GanSu, Lanzhou, 730050, China. \\ ${ }^{2}$ School of Design Art, LanZhou University of Technology Gansu, Lanzhou 730050, China
}

\begin{abstract}
An unsteady three-dimensional simulation based on Reynolds time-averaged control equations and RNG k- $\varepsilon$ turbulence model is presented for hydraulic turbines, and the pressure fluctuation and radial force characteristic of hydraulic turbines are obtained. The results obtained show that the time domain of pressure fluctuation for hydraulic turbine varies periodically, the greater the flow rate, the more serious the pressure fluctuation, the dominant frequencies of pressure fluctuation concentrate at low frequency region. After adding guide vane for hydraulic turbine, the pressure fluctuation in volute tongue reduces 30 percent, and under large flow rate condition, the maximum pressure fluctuation amplitude in dynamic and static coupling surface reduces $19 \%$, the dominant frequency is below the blade frequency of impeller; in high frequency region the pressure fluctuation decreases greatly. By adding guide vane, the radial force of turbine also can be greatly reduced.
\end{abstract}

Keywords: hydraulic turbine; guide vane; pressure fluctuation; radial force; numerical analysis.

\section{Introduction}

Centrifugal pump in reverse operation can be used as a turbine to recycle residual energy of high pressure fluid, and has a high efficiency[1-4], but in the process of running this kind of hydraulic turbine has some disadvantages, such as unsteady rotate speed, vibration, flow separation, etc, sometimes even can not meet the requirement of output. One of the key factors for above is that in turbine impeller inlet the pressure distribution is not uniform, which makes pressure fluctuation and radial force produced, Domestic and foreign many of researches on pressure fluctuation and radial force had been studied [ 5,6], but the research on pressure pulsation and radial force for hydraulic turbine with a guide vane is not much. in this paper, based on the pressure fluctuation characteristics of centrifugal pump, a guide vane is added for hydraulic turbine, and numerical simulation is used to analyse the pressure fluctuation and radial force, the effects of guide vane on pressure fluctuation and radial force are studied.

a Corresponding author : 584003101@qq.com

(c) 2016. The authors - Published by Atlantis Press 


\section{Calculation model and monitoring points}

Two calculation models are selected: one is a turbine without guide vane, the other is a turbine with a guide vane, the design parameter of two turbines are same : flow rate $q_{0}=62.79 \mathrm{~m}^{3} / \mathrm{h}$, head $\mathrm{h}=81 \mathrm{~m}$ and rotating speed $n=2900 \mathrm{r} / \mathrm{min}$. The geometric parameters for impeller: inlet diameter $\mathrm{d}_{2}=276 \mathrm{~mm}$, inlet width $b_{2}=6 \mathrm{~mm}$, outlet diameter $d_{1}=90 \mathrm{~mm}$, blade number $z=5$. on the basis of the first model , $a$ guide vane is added for the second model, the geometric parameters for guide vane: blade thickness $s$ $=4 \mathrm{~mm}$, blade numbers $z=6$, length of guide vane $l=30 \mathrm{~mm}$, the inlet angle and outlet angle of guide vane is $13^{\circ}$ and $9^{\circ}$. non-impact entrance is adopted for guide vane, but the diameter of base circle for second model is amplified, which is $\mathrm{d} 3=345 \mathrm{~mm}$, other dimensions are changeless. In order to roundly monitor the pressure fluctuation in flow field, totally 4 monitoring points (P1, P2, P4, P5) in dynamic and static coupling surface are selected, and the other point $\mathrm{p} 3$ is selected in volute tongue. All the monitoring points are shown in figure 1.

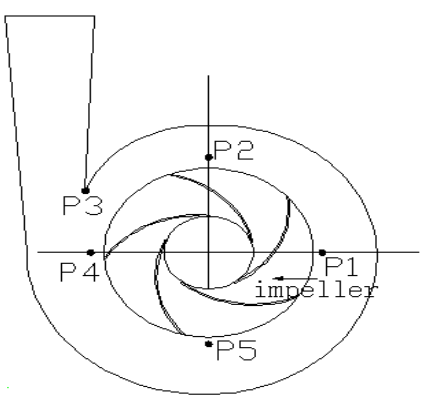

(a) model a

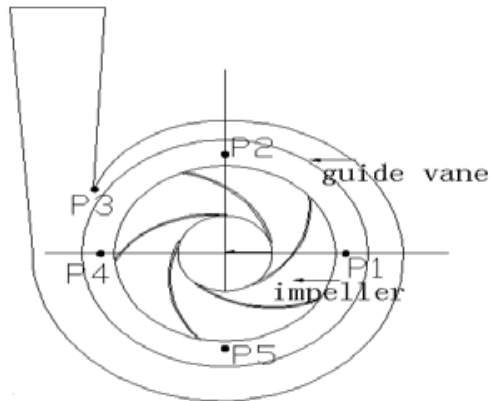

(b) model b

Figure 1. Monitoring points in hydraulic turbine

\subsection{Numerical solution method}

For the pressure fluctuation of centrifugal pump, the large eddy simulation approach and Reynolds time-averaged approach are applied mainly, but compared to the large eddy simulation, Reynolds time-averaged method is more mature, low requirements on the grid and computer, and short computing time needed [7].In this paper Reynolds time-averaged and $R N G \quad K-\varepsilon$ turbulent model are adopted. Unstructured grid is meshed by gambit software, and the grid cell numbers of model a and b is 453107 and 507508 respectively. After relativity checking, both equiangular slope and scale of grids in computational region are not more than 0.85. sliding mesh technology is performed inside the static and dynamic coupling interface, piso algorithm is adopted for the simultaneous solution of the momentum equation and continuity equation. Velocity conditions is used for the inlet boundary condition, pressure conditions is used for the outlet boundary condition. The calculation accuracy of convergence is $10^{-4}$, a complete calculation is done from inlet of volute to outlet of impeller. The time step of unsteady numerical simulation is defined $2.069 \times 10^{-4} / \mathrm{s}$, so the impeller rotates one circle per 100 time steps, and the impeller totally rotates four circles, the computation result of the fourth circle is adopted for analysis.

\section{Result and analysis}

\subsection{Characteristics of pressure fluctuation in hydraulic turbine}

The pressure fluctuation time domain curves of $\mathrm{p} 3$ for model a and model b under different conditions are shown in figure 2. Totally four conditions are simulated: 0.5 qe, qe, and 1.5 qe, qe is the flow rate of the best efficiency point. 

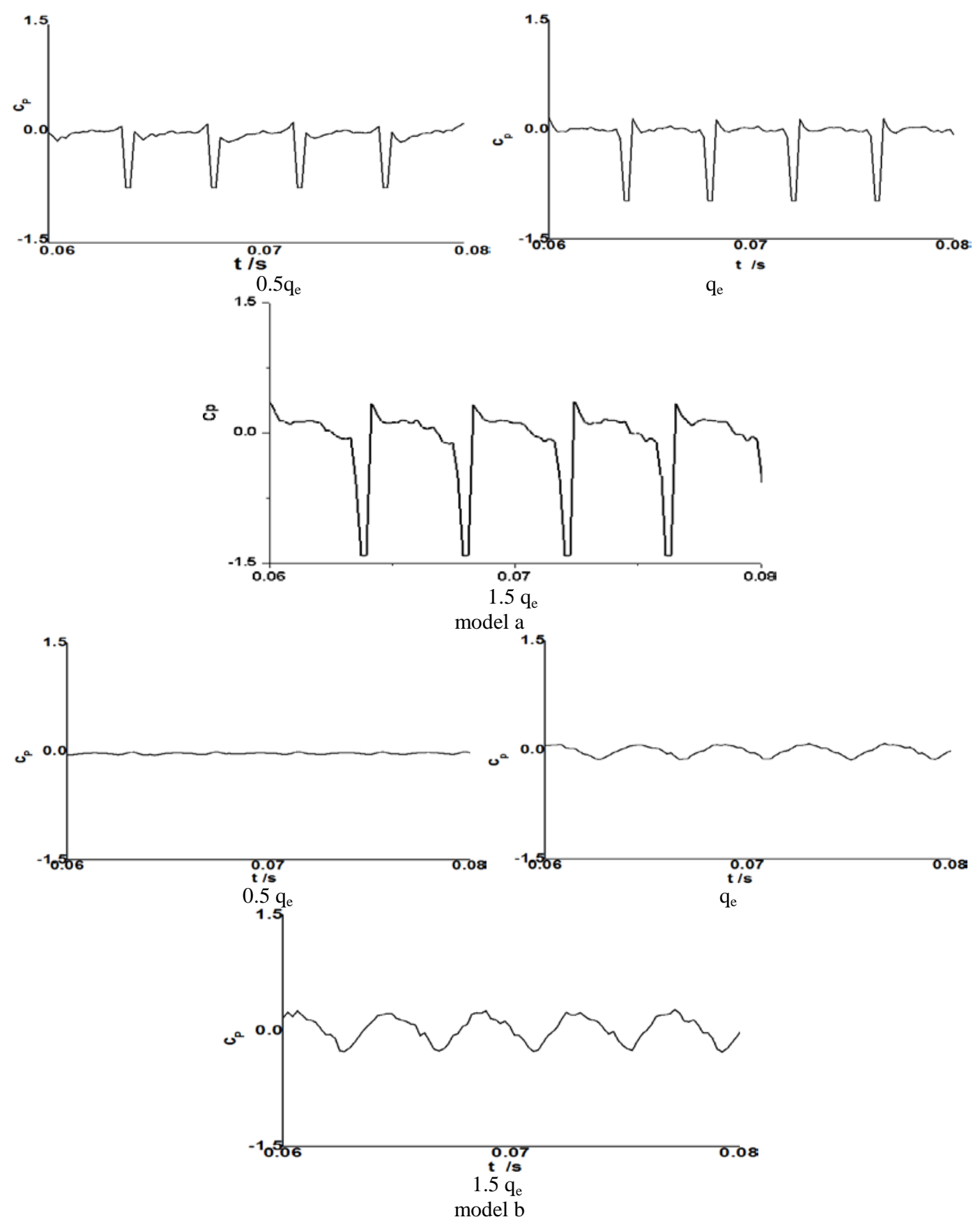

Figure 2. The pressure fluctuation time domain curve of p3 under different conditions

The abscissa represents time in one cycle, the ordinate represents coefficient of static pressures, and the ordinate pressure coefficient calculated as follow:

$$
c_{p}=\frac{\Delta p}{0.5 \rho u_{2}^{2}}
$$


$C_{p}$-Pressure coefficient,

$\Delta p$-Pressure difference of monitor point and average value75,

$u_{2}$-Peripheral speed of impeller inlet.

From figure 2: with different flow rate, time domain of pressure fluctuation changes periodically. under a small flow rate, the pressure fluctuation is light, under a large flow rate, the pressure fluctuation is serious. For a same time, the pressure fluctuation amplitude increases with the increase of flow rate. P3 locates at the volute tongue, the pressure coefficient value of model a is mainly negative, but the pressure coefficient of model $b$ has a uniform positive and negative distribution. For a same condition, the pressure coefficient curve of model b varies gently, even more, the absolute value of pressure coefficient for model $b$ is less than that of model $a$, the guide vane relieves the pressure fluctuation near the volute tongue. As is shown: the waveform of pressure fluctuation in the best efficiency condition is completely consistent with that of the large flow condition, the waveform of pressure pulsation in small flow rate condition is different, but the result is not affected, the reason is that when the pump in reverse operation as a turbine, the flow rate of turbine condition is greater than that of pump condition. the best efficiency point usually happens in large flow rate area, under small flow rate condition both the head and efficiency is more lower, which is not meet requirements.

The pressure fluctuation frequency domain characteristics curve of p3for model a and model b in different conditions is shown in figure 3. From figure 3: the dominant frequency of hydraulic turbine mainly concentrates at low frequency region, the pressure fluctuation decreases with the frequency increases. The amplitude of dominant frequency is associated with flow rate, with a small flow rate, the pressure fluctuation amplitude is small, while with a large flow rate, the pressure fluctuation amplitude is large. The greater the flow rate, the more influenced by frequency. Under 1.5Qe condition, both for model a and $\mathrm{b}$, the maximum pressure fluctuation amplitude happens at $\mathrm{f}=226.6 \mathrm{~Hz}$, and the corresponding pressure coefficient for model a is $2.5 \times 10^{-4}$, for model b is $1.74 \times 10^{-4}$. Compared with model a, pressure coefficient of model $b$ declines $30 \%$.So by adding a guide vane, the pressure fluctuation of turbine can be reduced.

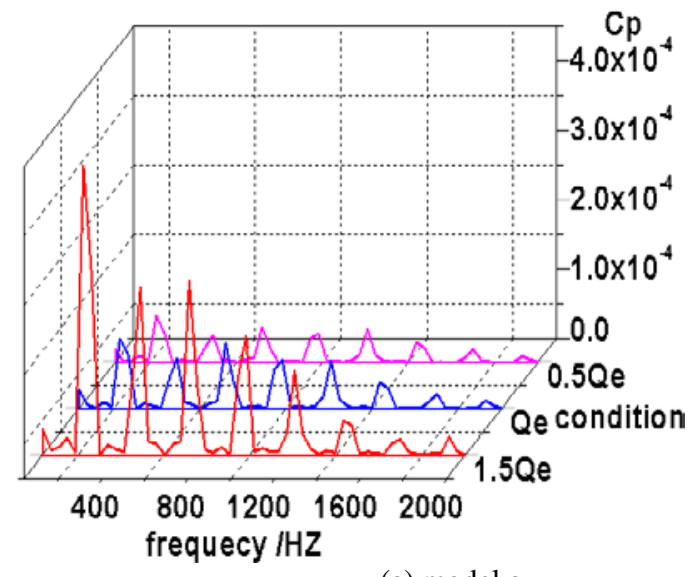

(a) model a

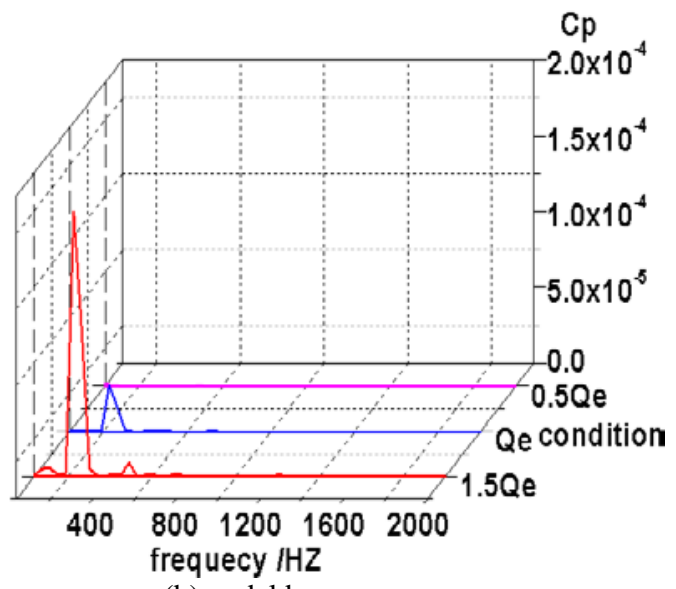

(b)model b

Figure 3. The frequency domain characteristics curve of pressure pulsation of $\mathrm{p} 3$ in different conditions

The pressure fluctuation time domain curves of p1, p2, p4, p5 under the optimal operating conditions are shown in figure 4 and figure5. p1, p2, p4 and p5 locate at the dynamic and static coupling surface. 

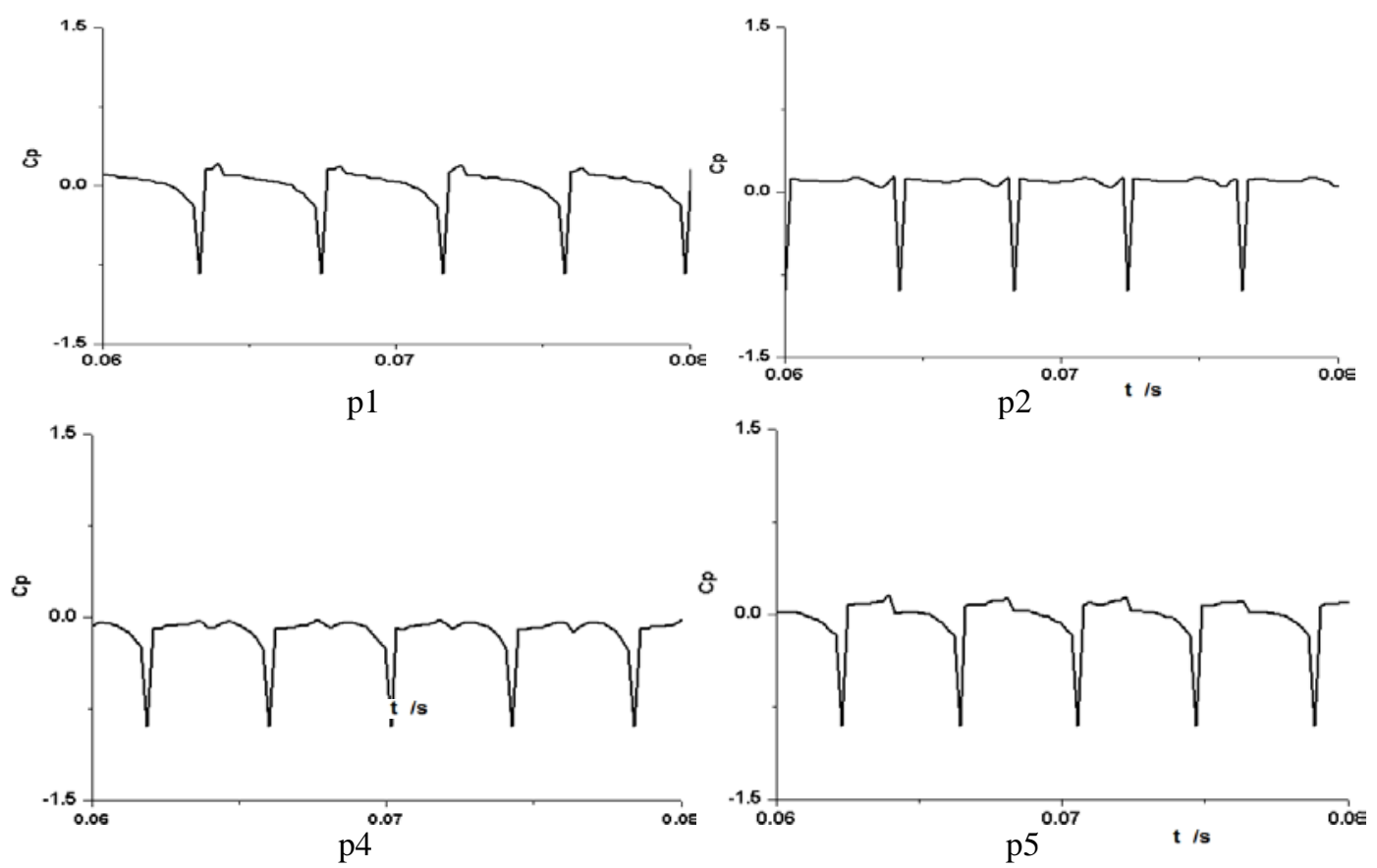

Figure 4. The pressure fluctuation time domain curves of p1, p2, p4, p5 for model a under the optimal operating conditions
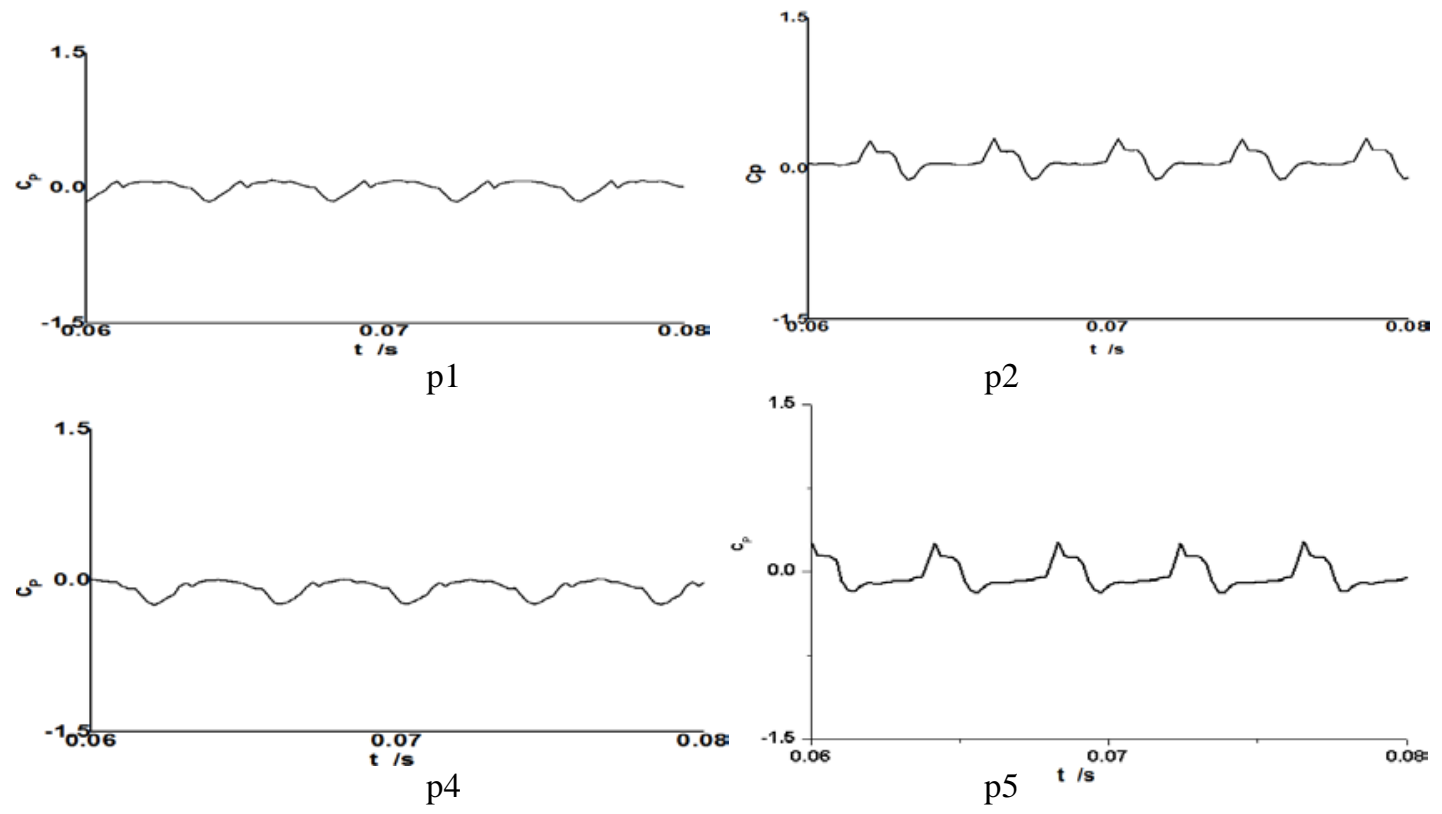

Figure 5. The pressure fluctuation time domain curves of p1, p2, p4, p5 for model b under the optimal operating conditions

From figure 4 and 5: the pressure fluctuation time domain of p1, p2, p4 and p5 show a periodic variation. in model a the waveform of pressure fluctuation for four monitoring points is similar, and the pressure coefficients are mainly negative, but in model B the waveform of pressure pulsation for $\mathrm{p} 1$ and p4 is similar, p2 and p5 is similar. While the phase difference of p1 and p4 is $180^{\circ}$, also p2 
and $\mathrm{p} 5$. The pressure coefficient of p1, p2, p4 andp5 for model b has a uniform positive and negative distribution, especially for monitoring point $\mathrm{p} 2$.

The pressure fluctuation frequency domain curves of p1, p2, p4, p5 for model a and b under the optimal operating conditions are shown in figure 6.

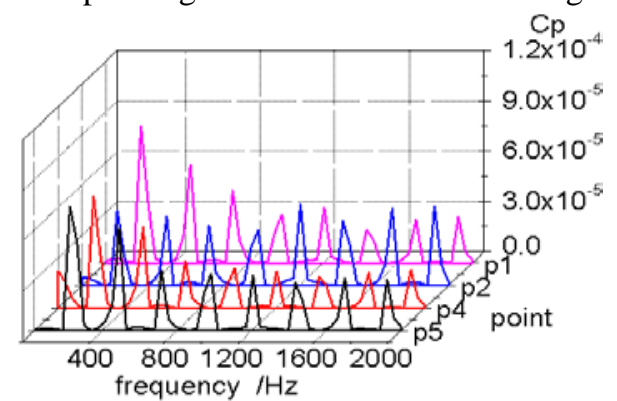

(a) model a

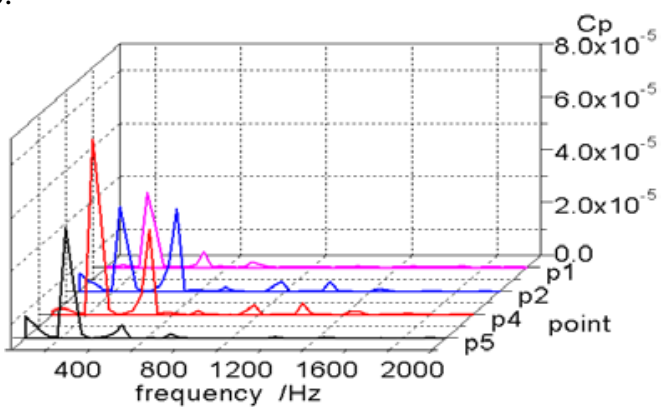

(b)model b

Figure 6. The pressure fluctuation time domain curves of p1, p2, p4, p5 under the optimal operating conditions

From figure 6: for model a the pressure fluctuation of four monitoring points exist universally in the low and high frequency region, and the monitoring point $\mathrm{p} 1$ is the most acute, the maximum pressure coefficient is $8.15 \times 10^{-5}$, the corresponding frequency $\mathrm{f}=226.6 \mathrm{~Hz}$, on the contrary the pressure fluctuation in monitoring point $\mathrm{p} 2$ is the most gentle. For model $\mathrm{b}$, the pressure fluctuation mainly concentrates in low frequency region. the pressure fluctuation in high frequency region is very small. the pressure fluctuation in monitoring point $\mathrm{p} 4$ is the most acute, the maximum pressure coefficient is $6.62 \times 10^{-5}$, and the corresponding frequency $\mathrm{f}=226.6 \mathrm{~Hz}$. Compared with model a, the maximum pressure pulsation amplitude of model b declines 19\%. When the turbine runner rotates along the counterclockwise, from the tongue, $\mathrm{p} 4$ is the first, followed by $\mathrm{p} 5, \mathrm{p} 1$, and p2, the pressure fluctuation decreases gradually along the rotating direction. As is shown: the dominant frequencies of maximum pressure fluctuation for model $a$ and $b$ happen in low frequency region, for the different monitor points, the pressure fluctuation amplitude are different, the pressure fluctuation decreases greatly by adding guide vane, the reason is as follow :after flowing through the volute, the liquid firstly flows into guide vane, then flows into impeller, which makes the liquid become more orderly and steady, and it is helpful to improve the operation stability for hydraulic turbine. For model a and b, The rotate speed $n=2900 \mathrm{r} / \mathrm{min}$, so the rotation frequency $\mathrm{f}=48.33 \mathrm{~Hz}$, the blade number $\mathrm{Z}=5$, so the blade frequency(product for frequency and number of blades) $\mathrm{f}=241.65 \mathrm{~Hz}$, the dominant frequencies for all monitor points $\mathrm{f}=226.6 \mathrm{~Hz}$, the dominant frequency of turbine is below the blade frequency.

\subsection{Radial force analysis}

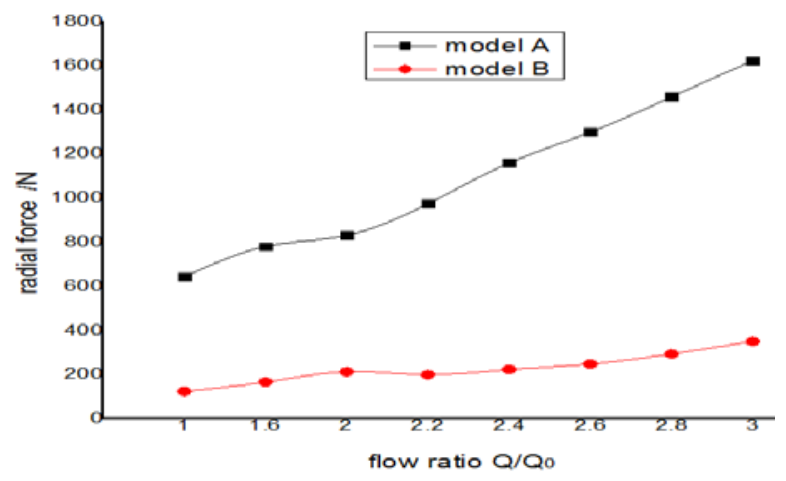

Figure 7. Relationship curve of flow ratio-radial force 
The relationship curve of flow ratio-radial force is shown in figure 7 . The hydraulic turbines are different from pumps, even under design condition, the radial force still exists. The reason is that the pressure distribution in volute is destroyed by some factors, such as unsteady flow in volute, design error and testing, etc. it is shown: the radial force increases with the flow rate increases, and approximately linear distribution, but the radial force of model $b$ is far less than that of model $a$, it is because the model $\mathrm{b}$ is added a guide vane, the guide vane is axially symmetric distribution in circumferential direction, it has a very good diversion effect, which makes the liquid velocity and pressure distribution more uniform, this result is in complete accord with reference [8].

\section{Conclusion}

The pressure fluctuation of hydraulic turbines shows a periodic variation. For a turbine without guide vane, The dominant frequency of pressure fluctuation concentrates at low frequency and some high frequency regions, but for a turbine added guide vane, the dominant frequency only is below the blade frequency, and the pressure fluctuation weakened greatly, especially in high frequency region, therefore, the pressure fluctuation can be reduced by adding guide vane, meanwhile, the radial force can be reduced greatly.

\section{Reference}

1. Shahram Derakhshan, Ahmad Nourbakhsh. Theoretical, numerical and experimental investigation of centrifugal pumps in reverse operation, J. Experimental Thermal and Fluid Science. 32(2008) 1620-1627.

2. J Ferna'ndez, E Blanco, J Parrondo, M T Stickland and T J Scanlon. Performance of a centrifugal pump running in inverse mode,J. Journal of power and energy, 18(2010) 265-271.

3. H.J. van Antwerpen , G.P. Greyvenstein. Use of turbines for simultaneous pressure regulation and recovery in secondary cooling water systems in deep mines,J. Energy Conversion and Management, 46 (2005) 563-575.

4. Pradeep Bansal. Nick Marshall. Feasibility of hydraulic power from waste energy in bio-gas scrubbing process,J. Applied Energy, 87 (2010) 1048-1053.

5. Yang Sun-Sheng, Kong Fan-Yu, Fu-Hui and Xue Ling, Numerical Research on Effect of Splitter Blades to the Influence of Pump as Turbine. International journal of Rotating Machinery, 2012

6. Cavazzini, Giovanna. Analysis of the unstable behavior of a pump-turbine in turbine mode: Fluid-dynamical andspectral characterization of the s-shape characteristic,J. Journal of Fluids Engineering, Transactions of the ASME, , 138(2016).

7. Zhu RongSheng, $\mathrm{Hu}$ Ziqiang, fu Qiang. Numerical simulation of pressure fluctuation in double-balde pump,J. Transactions of the CSAE J,26 (2010) 129-134.(in Chinese)

8. G Ventrone, $G$ Ardizzon and $G$ Pavesi. Direct and reverse flow conditions in radial flow hydraulic turbomachines,J. Proc Instn Mech Engrs, 214, Part A:635-644. 